\title{
Design of a Pierce Oscillator For CMOS SAW Resonator
}

\author{
Jamilah Karim \\ Electrical and Computer Engineering Department \\ Faculty of Engineering \\ IIUM, Gombak. \\ Electrical Engineering Faculty \\ University Technology MARA \\ Shah Alam, Selangor
}

\author{
Anis Nurashikin Nordin, AHM Zahirul Alam \\ Electrical and Computer Engineering Department \\ Faculty of Engineering \\ IIUM, Gombak
}

\begin{abstract}
Development of microelectromechanical system (MEMS) based oscillators have drawn significant attention because it provides CMOS compatibility and multifrequency operations on a single chip. Recently, integrated MEMS resonators have shown great performance by attaining high quality factors and high frequency operations of up to the $\mathbf{G H z}$ range. Of interest, is fully integrated $\mathrm{SAW}$ resonator which can be connected to an oscillator circuit on the same chip. For oscillator circuit simulations, the CMOS SAW resonator was modeled using its RLC equivalent circuits. The insertion loss of CMOS SAW resonator used in this design is $35.8 \mathrm{~dB}$, with motional resistance $R x=8.95 \mathrm{k} \Omega$ and the motional capacitance and inductance are $\mathrm{Cx}=199 \mathrm{aF}$ and $\mathrm{Lx}=350 \mathrm{uH}$. For a MEMS resonator to be able to function as an oscillator it needs to be coupled with supporting circuits. There are various types of supporting oscillator circuit topologies namely the pierce oscillator, differential amplifier oscillator or the transimpedance amplifier circuit topology. The topology to be chosen depends on the design requirement, the loop gain of 1 and the zero phase shifts. For this work, the pierce circuit topology was chosen due to its simplicity and high frequency stability. This simple circuit comprising of 4 transistors, helps to achieve low power consumption and excellent phase noise characteristics. This paper will present the analysis, design and the simulation result of a high gain $(>36 \mathrm{~dB})$ and low power pierce circuit topology for MEMS CMOS SAW resonator. The circuit was designed in $0.18 \mathrm{um}$ CMOS technology and yield open loop gain $>36 \mathrm{~dB}$.
\end{abstract}

Keywords- CMOS MEMS Oscillator, Pierce Oscillator, MEMS $S A W$ resonator

\section{INTRODUCTION}

The quest for low power, low cost and small size solution in wireless communication system has triggered a desire to implement full integrated system. As such the development of MEMS based oscillator has drawn significant attention because it has provided great promising for fully integration system. MEMS based oscillators have demonstrated high frequency resonant (in $\mathrm{GHz}$ range), low power and small size. However, it still needs a lot of improvement in terms of reliability, frequency stability, low phase noise and low power consumption to meet the requirements of communication systems. Current achievement of MEMS based oscillators reported are i) at oscillation frequency $1.05 \mathrm{GHz}$, phase noise performance was $-81 \mathrm{dBc} / \mathrm{Hz}$ at $1 \mathrm{kHz}$ and consume $3.5 \mathrm{~mW}$ power [1], ii) [2] has demonstrated $1.9 \mathrm{GHz}$ oscillator that consume $0.3 \mathrm{~mW}$ power with phase noise performance of $-100 \mathrm{dBc} / \mathrm{Hz}$ at $10 \mathrm{kHz}$, iii) [3] has reported a $1.006 \mathrm{GHz}$ oscillator with phase noise $-119 \mathrm{dBc} / \mathrm{Hz}$ at $10 \mathrm{kHz}$ and consume about $7.2 \mathrm{~mW}$ power and iv) $8.3 \mathrm{MHz}$ oscillator was reported in [4] with phase noise performance about $-89 \mathrm{dBc} / \mathrm{Hz}$ at $1 \mathrm{kHz}$ and $8.7 \mathrm{~mW}$ power.

The key component of MEMS based oscillators are the amplifier and the frequency determination, which is MEMS resonator. These two components are connected in feedback connection. This paper will report about the designed amplifier to be integrated with MEMS SAW resonator. The details about the resonator used is presented in section II, followed by the design process in section III, the simulation result is shown in section IV and section $\mathrm{V}$ is the conclusion of the work done.

\section{CMOS MEMS SAW RESONATOR}

Surface Acoustic Wave(SAW) devices has been used since ages as a frequency determining element and filter in RF applications because they have powerfull signal processing capabilities and easily manufactured. Later with the advancement in CMOS technology and due to its popularity in RFIC field has motivated [5] to produce CMOS MEMS SAW resonator. This resonator comprise numerous advantages such as i) can directly oscillate up to several $\mathrm{GHz}$ frequency without the need of any extra circuitry, ii) good phase noise performance, iii) more robust and provide wide range of application[6] and iv) low power frequency generator. This MEMS SAW resonator can be used as an RF oscillator and has been approved by [7].

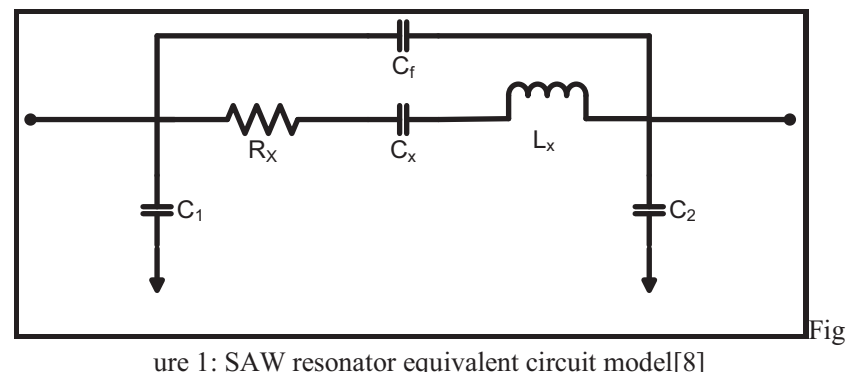

ure 1: SAW resonator equivalent circuit model[8] 
Fig 1, shows the equivalent circuit model of a SAW resonator and the cross section is shown in fig 2 . The parameter of each components in the model are $\mathrm{R}_{\mathrm{x}}=8.95 \mathrm{k} \Omega, \mathrm{C}_{\mathrm{x}}=199 \mathrm{aF}$, $\mathrm{L}_{\mathrm{x}}=350 \mathrm{uH}, \mathrm{C}_{\mathrm{f}}=24.9 \mathrm{fF}, \mathrm{C}_{1}=\mathrm{C}_{2}=0.206 \mathrm{pF}$. These parameter were obtained from[5]. The resonant frequencies established by this resonator are $\mathrm{f}_{\mathrm{s}}=601.9 \mathrm{MHz}$ and $\mathrm{f}_{\mathrm{p}}=606.6 \mathrm{MHz}$ with insertion $\operatorname{loss}(\mathrm{IL})=36 \mathrm{~dB}$.

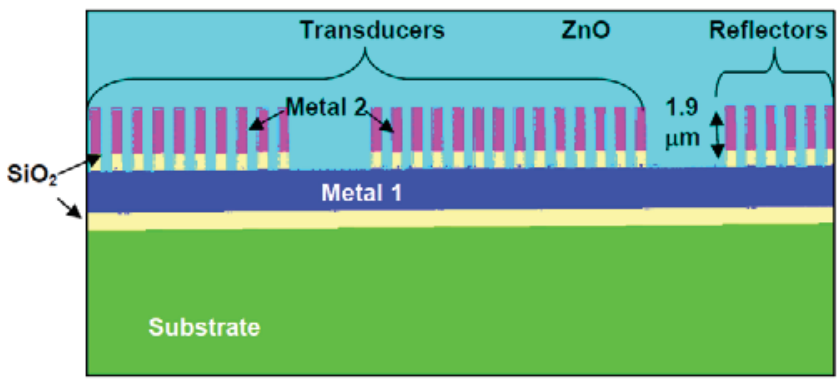

Figure 2: The Cross-section of CMOS SAW resonator utilizing two CMOS metal layers

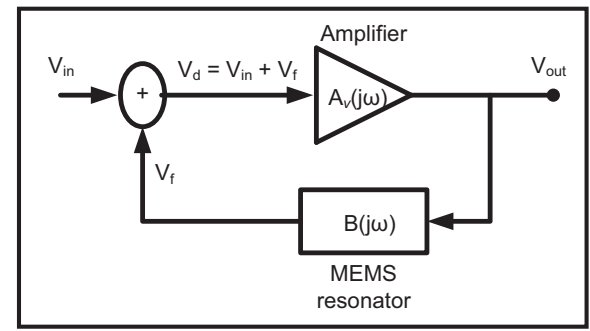

Figure 2: Basic Feedback Oscillator Circuit

\section{AMPLIFIER CIRCUIT DESIGN}

Fig 2 is a block diagram of basic feedback oscillator circuit. This circuit consists of an amplifier and the MEMS SAW resonator. The amplifier chosen to be used in an oscillator circuit must be able to overcome the insertion losses of the resonator. The oscillator circuit also has to have a unity loop gain and zero phase shift as shown in the equation below so that the oscillation can be started and sustained.

The close loop gain obtained from Fig 2 is shown in equation 1 .

$$
A_{v f}=\frac{A_{v}}{1-\beta A_{v}}
$$

From 1, the loop gain is:

$$
\left|\beta A_{v}\right| \geq 1
$$

And the phase shift is:

$$
\begin{gathered}
\angle \beta A_{v}= \pm 360^{\circ} \\
n=0,1,2,3, \ldots \ldots \ldots \ldots \ldots \ldots
\end{gathered}
$$

And gain for the amplifier is:

$$
A_{v}=g_{m 1} R_{L} \frac{C_{2}}{C_{1}}
$$

The design will be implemented using 0.18um CMOS technology. The pierce oscillator circuit topology was chosen, since this topology is simple and provides straightforward biasing. This topology also has superior performance in terms of low power consumption and phase noise characteristics.

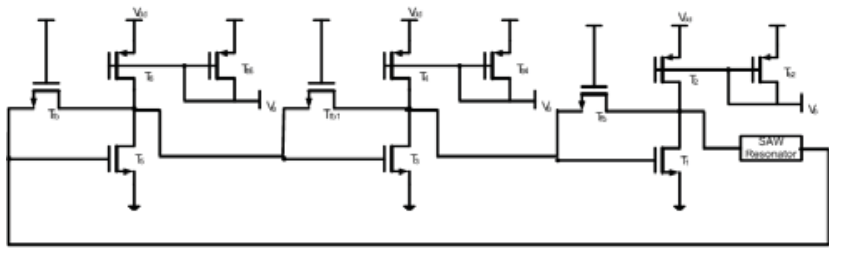

Figure 3: Transistor level of pierce oscillator circuit topology[2]

The transistor level of pierce oscillator circuit topology is shown in Fig 3. Three stage of amplifier was needed in order to provide sufficient gain to eliminate the losses of the resonator. Transistor $\mathrm{T}_{1}, \mathrm{~T}_{3}$ and $\mathrm{T}_{5}$ provides the critical transconductance for oscillation. The MEMS SAW resonator is connected across the drain and gate of this device. $\mathrm{T}_{\mathrm{fb}}, \mathrm{T}_{\mathrm{fb} 1}$ and $\mathrm{T}_{\mathrm{fb} 2}$ provide a large bias resistor to the gate of $T_{1}, T_{3}$ and $T_{5}$ respectively. The bias current is supplied by $T_{2}, T_{4}$ and $T_{6}$ of every respective stage.

The amplifier is biased in weak inversion region. In weak inversion region the current dominates by diffusion current and drain current is proportional to the exponential gate-source votage, $\mathrm{V}_{\mathrm{Gs}}$. CMOS operated in weak inversion region requires large shape factor, $S=W / L$, thus result in large gate area, high gate capacitance and poor circuit bandwidth. Besides all the weaknesses, weak to moderates inversion region carries more advantages like high $\mathrm{DC}$ gain, minimum $\mathrm{V}_{\mathrm{GS}}$, higher $\mathrm{g}_{\mathrm{m}}$, minimum $\mathrm{V}_{\mathrm{DSAT}}$ and very important method for low power and low voltage circuit design.

The transistor used in this circuit topology is design to bias in weak inversion region since the estimated critical transconductance of transistor is high, $\mathrm{g}_{\mathrm{m}}=13.1 \mathrm{mS}$. This value was obtained from the following equation:

$$
g_{m} \geq \omega_{0}^{2}\left(C_{f}+\frac{C_{1} C_{2}}{C_{1}+C_{2}}\right)^{2}\left(\frac{\left(C_{1}+C_{2}\right)^{2}}{C_{2}^{2}}\right)\left(R_{p}\right)
$$

And $R_{p}=R_{x}+R_{\text {cap }}$ at resonant frequency.

The amplifier operated at $\mathrm{V}_{\mathrm{dd}}=1.8 \mathrm{~V}, \quad \mathrm{~V}_{\mathrm{b}}=500 \mathrm{mV}$, $\mathrm{I}_{\mathrm{d} 1}=92.52 \mathrm{uA}, \quad \mathrm{I}_{\mathrm{d} 3}=670.23 \mathrm{uA}$ and $\mathrm{I}_{\mathrm{d} 5}=675.92 \mathrm{uA}$, and the transconductance for this three main transistors are $\mathrm{g}_{\mathrm{m} 1}=1.95 \mathrm{mS}, \mathrm{g}_{\mathrm{m} 3}=13.51 \mathrm{mS}$ and $\mathrm{g}_{\mathrm{m} 5}=13.61 \mathrm{mS}$.

\section{RESULT AND DISCUSSION}

The simulated admittance plots (S21) for MEMS SAW resonator is shown in Fig 4. The series resonant frequency obtained from this simulation is $\mathrm{f}_{\mathrm{s}}=604.2 \mathrm{MHz}$ and series impedance $R_{s}=67.8 \Omega$ or $I L=36.6 \mathrm{~dB}$. The parallel resonant frequency $f_{p}=609.3 \mathrm{MHz}$ and the parallel impedance 
$R_{p}=246.8 \Omega$ or $I L=47.8 \mathrm{~dB}$. Thus the designed amplifier must have gain $A_{v} \geq 36 \mathrm{~dB}$.

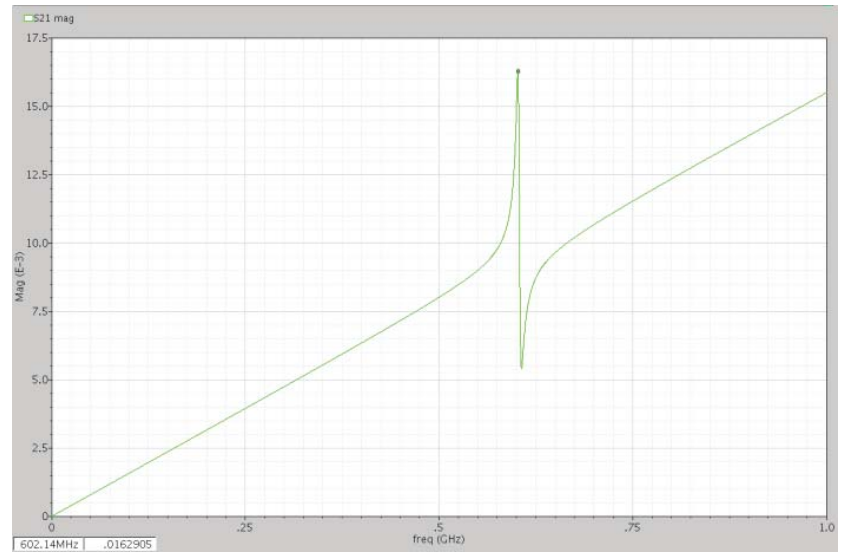

(a)

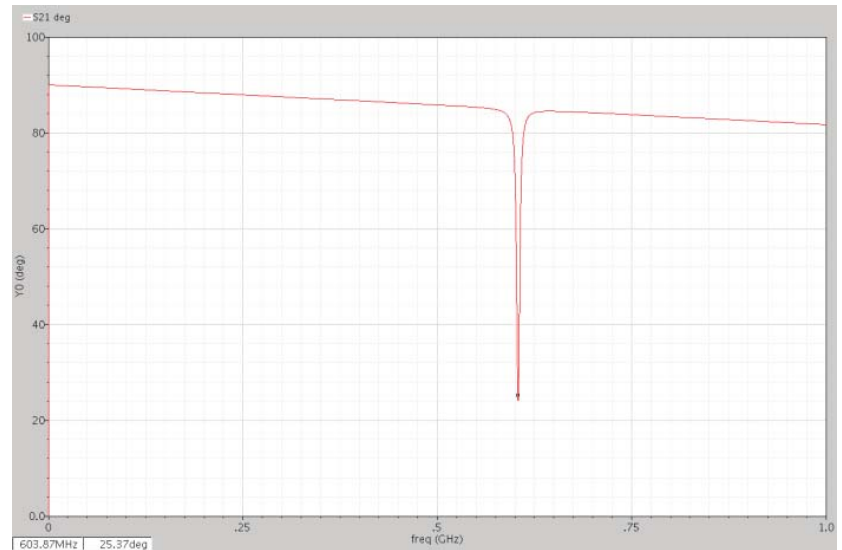

(b)

Figure 4: Simulated admittance plot for $602 \mathrm{MHz}$ resonator (a) Magnitude (b) Phase

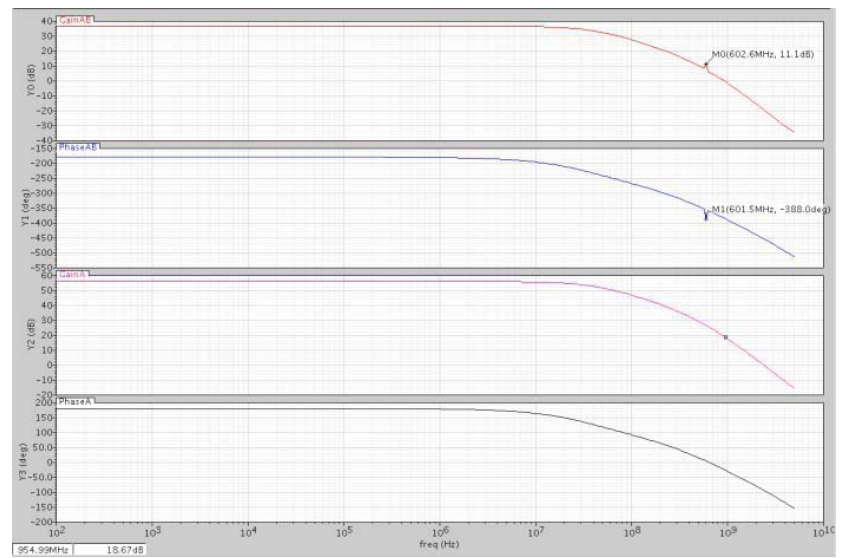

Figure 5: Simulated result for Gain and Phase of the amplifier (GainA and Phase $\mathrm{A}$ ) and amplifier with resonator (Gain $\mathrm{AB}$ and Phase $\mathrm{AB}$ ).
Fig 5 has shown the simulation result of the amplifier gain and phase, and also the simulation result of the gain and phase when the resonator was connected to the amplifier. The parameter of the transistor used to build the amplifier are as follows: $\mathrm{T}_{1}=100 \mathrm{u} / 0.18 \mathrm{u}, \mathrm{T}_{2}=\mathrm{T}_{\mathrm{b} 2}=4.5 \mathrm{u} / 1 \mathrm{u}, \mathrm{T}_{3}=\mathrm{T}_{5}=250 \mathrm{u} / 0.18 \mathrm{u}$, $\mathrm{T}_{4}=\mathrm{T}_{\mathrm{b} 4}=\mathrm{T}_{6}=\mathrm{T}_{\mathrm{b} 6}=4.5 \mathrm{u} / 0.18 \mathrm{u}, \mathrm{T}_{\mathrm{fb}}=\mathrm{T}_{\mathrm{fb} 1}=0.22 \mathrm{u} / 4 \mathrm{u}$. The topology yields the open loop gain and phase, $A_{v}=56 \mathrm{~dB},\left\llcorner A_{v}=180^{\circ}\right.$, $A_{v} \beta=11.1 \mathrm{~d} B$ and $\left\llcorner A_{v} \beta=-388^{\circ}\right.$.

The oscillation frequency produced after the closed loop simulation was $609 \mathrm{MHz}$ with oscillation voltage about $51.2 \mathrm{mV}$ as shown in fig 6 . The phase noise performance was shown in fig 7. This oscillator has achieved the phase noise of about $170 \mathrm{dBc} / \mathrm{Hz}$ at $251.2 \mathrm{~Hz}$.

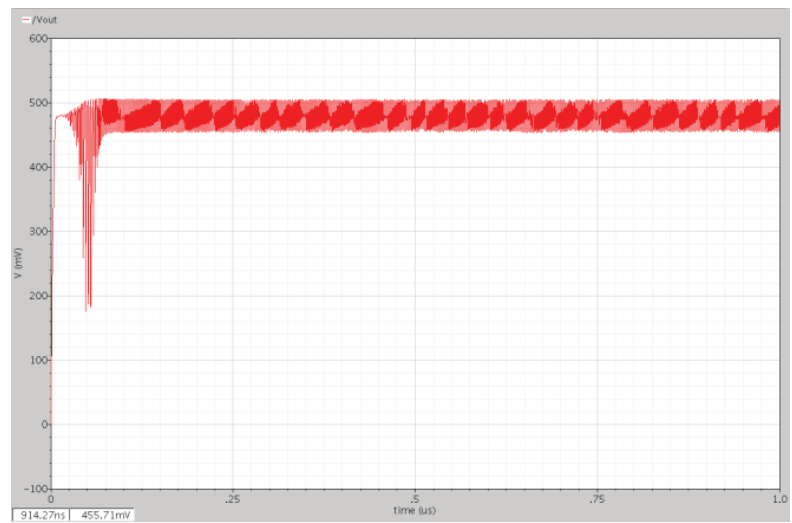

Figure 6: Simulated frequency Oscillation of CMOS MEMS SAW oscillator

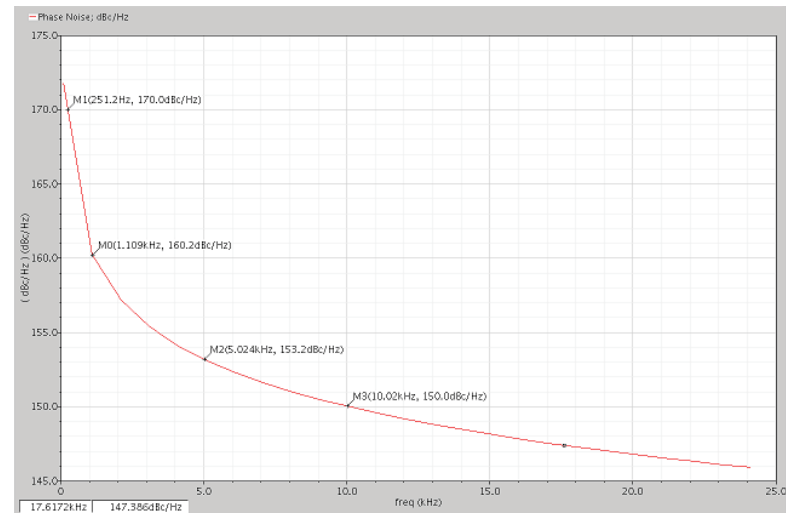

Figure 7: Simulated Phase Noise of CMOS MEMS SAW oscillator

\section{CONCLUSION}

The topology has been designed and simulated. From simulation made, this topology can achieve the required gain to overcome the insertion losses of the resonator, and yield $609 \mathrm{MHz}$ frequency oscillation with phase noise performance about 170dBc/Hz@251Hz. Further improvement is needed for this CMOS MEMS SAW oscillator in terms of its oscillation frequency and phase noise performance. 


\section{REFERENCES}

[1] Z. Chengjie, J. Van Der Spiegel, and G. Piazza, "1.05-GHz CMOS oscillator based on lateral- field-excited piezoelectric AIN contourmode MEMS resonators," Ultrasonics, Ferroelectrics and Frequency Control, IEEE Transactions on, vol. 57, pp. 82-87, 2010.

[2] B. P. Otis, "The Design and Implementation of an Ultra Low Power RF Oscillator Using Micromachined Resonators ". vol. Master of Science, Plan II Berkeley: Department of Electrical Engineering and Computer Sciences, University of California p. 82.

[3] H. M. Lavasani, P. Wanling, B. Harrington, R. Abdolvand, and F. Ayazi, "A 76 dB, $1.7 \mathrm{GHz}$ 0.18um CMOS Tunable TIA Using Broadband Current Pre-Amplifier for High Frequency Lateral MEMS Oscillators," Solid-State Circuits, IEEE Journal of, vol. 46, pp. 224-235, 2011.

[4] F. Nabki, K. Allidina, F. Ahmad, P. V. Cicek, and M. N. El-Gamal, "A Highly Integrated $1.8 \mathrm{GHz}$ Frequency Synthesizer Based on a MEMS Resonator," Solid-State Circuits, IEEE Journal of, vol. 44, pp. 2154$2168,2009$.

[5] A. N. Nordin, "Design, Implementation and Characterization of Temperature Compensated SAW Resonators in CMOS Technology for RF Oscillators " in School of Engineering and Applied Science: The george Washington University, 2008, p. 221.

[6] R. Aigner, "SAW and BAW technologies for RF filter applications: A review of the relative strengths and weaknesses," in Ultrasonics Symposium, 2008. IUS 2008. IEEE, 2008, pp. 582-589.

[7] A. Nordin and M. Zaghloul, "RF oscillator implementation using integrated CMOS surface acoustic wave resonators," Analog Integrated Circuits and Signal Processing, pp. 1-10, 8 february 20112011.

[8] "IEEE Standard Definitions and Methods of Measurement for Piezoelectric Vibrators," IEEE Std No.177, p. 1, 1966. 